\title{
Diversidade e uso do ambiente pelos anfíbios e répteis da Ilha de São Sebastião, Ilhabela, SP
}

Dissertação apresentada ao Programa de PósGraduação Interunidades em Biotecnologia USP/Instituto Butantan/IPT da Universidade de São Paulo, para obtenção do Título de Mestre em Biotecnologia.

Área de Concentração: Biotecnologia

Orientador: Prof. Dr. Ricardo J. Sawaya

São Paulo 


\section{RESUMO}

Centeno FC. Diversidade e uso do ambiente pelos anfíbios e répteis da Ilha de São Sebastião, Ilhabela, SP [dissertação]. São Paulo: Instituto de Ciências Biomédicas da Universidade de São Paulo; 2008.

A Mata Atlântica é um dos biomas com maior diversidade de espécies, considerada um dos hotspots mundiais de biodiversidade mais ameaçados. Atualmente, está restrita a 7,6\% de sua extensão original, e é na Serra do Mar que se encontra a parcela mais representativa. A Ilha de São Sebastião está localizada neste remanescente de Mata Atlântica e, apesar de sua importância, o conhecimento sobre as taxocenoses da herpetofauna ainda é fragmentado, principalmente no que diz respeito à ecologia. Assim, considerando a inexistência de dados ou trabalhos quantitativos, este estudo teve como objetivo o estudo da ecologia da herpetofauna da Ilha de São Sebastião, enfocando os seguintes aspectos: composição, riqueza, dominância, abundância relativa de espécies, uso do ambiente e uso do substrato. Os dados foram obtidos durante 101 dias de trabalho de campo, entre dezembro de 2005 e julho de 2007. Para a amostragem de campo, foram utilizados os métodos armadilha de interceptação e queda, armadilha de funil, procura visual limitada por tempo, procura de carro e encontro casual. Os dados de campo foram complementados por dados de coleções científicas e literatura. Foram registradas, no total, 34 espécies de anfíbios anuros, uma de anfíbio gimnofiono, um anfisbenídeo, dez lagartos, 26 serpentes e um quelônio. Os cinco métodos de amostragem possibilitaram a captura de 1.082 indivíduos, sendo 842 anfíbios e 240 répteis; e um total de 50 espécies, sendo 27 anfíbios anuros, um anfíbio gimnofiono, um anfisbenídeo, cinco lagartos, 21 serpentes e um quelônio. O método responsável pela captura do maior número de indivíduos foi armadilha de interceptação e queda, e os métodos que proporcionaram o registro do maior número de espécies foram procura visual limitada por tempo e encontro casual para anfíbios anuros, e armadilha de interceptação e queda para répteis Squamata. A ilha apresenta maior número de espécies raras do que comuns. A espécie de anuro dominante foi Leptodactylus cf. marmoratus, com 30,8\% do total de anuros capturados. Bothrops jararaca foi a espécie dominante entre os répteis Squamata, com 29,5\% do número total de indivíduos. O modo como as espécies utilizam o substrato é bastante diversificado, sendo que serapilheira, arbusto e solo foram os substratos mais utilizados pela herpetofauna. A maioria das espécies é especialista no uso do substrato, porém, foi observado número relativamente alto de 
espécies com habitat preferencial ou opcional. Quanto ao uso do ambiente, a distribuição das espécies indica que ocorre estruturação das taxocenoses, com maior diversidade na altitude intermediária. Os resultados obtidos refletem a grande diversidade de espécies existente na Mata Atlântica, evidenciando o grande potencial da Ilha de São Sebastião para estudos sobre ecologia e história natural da herpetofauna. Assim, a Ilha de São Sebastião é de extrema importância para a conservação por abrigar uma fauna rica e diversificada, incluindo uma espécie endêmica (Siphonops insulanus) e três ameaçadas de extinção (Chiasmocleis carvalhoi, Mabuya caissara e Stereocyclops parkeri).

Palavras-chave: Diversidade. Uso do substrato. Uso do ambiente. Herpetofauna. Ilha de São Sebastião. Mata Atlântica. 
Centeno FC. Diversity and habitat use by amphibians and reptiles from Ilha de São Sebastião, Ilhabela, SP [master thesis]. São Paulo: Instituto de Ciências Biomédicas da Universidade de São Paulo; 2008.

The Atlantic Forest is among the most diverse biomes, considered as one of the hotspots in the world. Nowadays, is restricted to $7.6 \%$ of its original extension, and the most representative area is in the Serra do Mar. The Ilha de São Sebastião is located in one of the most representative remnants of the Atlantic Forest, and although its great importance, the knowledge of herpetofaunal assemblages remain poorly known, mainly in ecology. Thus, considering the data or quantitative studies absence, this work goal the herpetofaunal ecology of Ilha de São Sebastião, focusing: composition, richness, dominance, relative abundance, habitat use and macrobabitat use. The data were obtained from 101 days of fieldwork, from December 2005 to July 2007. Fieldwork included pitfall traps with drift fences, funnel traps, time constrained search, sampling by car and incidental encounters. The fieldwork data was complemented by herpetological collections and literature records. In the totality, we recorded 34 species of anuran amphibians, one gymnophiona, one amphisbenid, ten lizards, 26 snakes, and one turtle. A total of 1.082 specimens were captured by the five sampling methods, among 842 amphibians and 240 reptiles; and 50 species that included 27 anurans, one gymnophiona, one amphisbenids, five lizards, 21 snakes, and one turtle. The method responsible for the higher number of individuals captured was pitfall traps with drift fences, and the methods that allowed the record of the higher number of species were time constrained search and incidental encounters for anurans and pitfall traps with drift fences for Squamata. The island shows a higher number of rare species. The dominant species of anurans was Leptodactylus cf. marmoratus, with $30.8 \%$ of the total number of frogs found. Bothrops jararaca was the dominant reptile species, with $29.5 \%$ of the total number of individuals. There were many differences on macrohabitat use, and the main were litter, shrub and ground. The most of species is specialist in the macrohabitat use, but, we observed relatively high number of species with preferential and optional habitat. In the habitat use, the species distribution shows that the assemblage structure is complex, with superior diversity in the intermediate altitude. This result reflects the high diversity of species in the Atlantic Forest, evidencing the great potential of Ilha de 
São Sebastião for studies on ecology and herpetofaunal natural history. Thus, the Ilha de São Sebastião is of extreme importance to conservation due to harbor a rich and diversified fauna, including one endemic species (Siphonops insulanus) and three threatened species (Chiasmocleis carvalhoi, Mabuya caissara and Stereocyclops parkeri).

Key words: Diversity. Macrohabitat use. Habitat use. Herpetofauna. Ilha de São Sebastião. Atlantic Forest. 


\section{INTRODUÇÃO GERAL}

A composição e diversidade de espécies são componentes básicos da estrutura de uma comunidade ou taxocenose, que inclui, entre outros aspectos, a distribuição ao longo do espaço e do tempo (Cadle, Greene, 1993; Ricklefs, 1990; Pough et al., 2004). Entretanto, informações básicas sobre ecologia de comunidades ainda são fragmentadas, apesar da importância e urgência da quantificação da diversidade biológica.

A região neotropical abriga grande parte da riqueza de anfíbios e répteis do planeta, sendo ideal para o estudo de padrões de diversidade (Cadle, Patton, 1988; Duellman, 1988; Heyer, 1988). Cerca de 80\% de todas as espécies existentes ocorre em áreas tropicais (Pough et al., 2004). Entretanto, a ecologia da herpetofauna dessas regiões ainda é relativamente pouco conhecida. Considerando os diversos estudos realizados na América do Sul, certamente a Mata Atlântica no sudeste do Brasil é a menos conhecida neste sentido (Marques, 1998). Alguns estudos que enfocam a diversidade dos anfíbios e répteis nesse bioma foram desenvolvidos nas últimas décadas (e.g. Heyer et al., 1990; Haddad, Sazima, 1992; Sazima, Haddad, 1992; Sawaya, 1999; Bertoluci, 2001; Marques et al., 2001; Sazima, 2001; Hartmann, 2004; Marques, Sazima, 2004; Pombal, Gordo, 2004; Hartmann, 2005; Dixo, Verdade, 2006; Cicchi, 2007; Cicchi et al., 2007; Moraes et al., 2007; Sena, 2007; Haddad et al., 2008), mas inventários adicionais e trabalhos detalhados sobre a ecologia das espécies e comunidades ainda são necessários.

A Mata Atlântica é considerada um dos biomas com maior diversidade e grande número de espécies endêmicas (Lino, 1992). Entretanto, apesar da grande riqueza de espécies e alto endemismo, aspectos básicos sobre a sua herpetofauna ainda são desconhecidos. Como resultado de 500 anos de destruição, os remanescentes de Mata Atlântica encontram-se sob intensa pressão antrópica e iminente risco de extinção (Morellato, Haddad, 2000). Atualmente, a Mata Atlântica está restrita a 98.800 km² remanescentes ou 7,6\% de sua extensão original [Ministério do Meio Ambiente (MMA), 1999], e é na Serra do Mar que se encontra a parcela mais representativa do que restou com aproximadamente $6 \%$ de sua cobertura vegetal no litoral, incluindo os ambientes insulares (SOS Mata Atlântica, 2002).

Pesquisas sobre estruturação de comunidades insulares são fundamentais para o desenvolvimento de teorias sobre ecologia e evolução (Vitousek et al., 1995). 
Ecossistemas insulares são ambientes propícios para a especiação, processo que depende de fatores como o grau de isolamento e o tempo de estabelecimento das populações ancestrais (Darwin, 1859; Carbonari, 1981). Assim, duas populações separadas por alguma barreira geográfica podem sofrer diferenciação ao longo do tempo, tornando-se espécies distintas (Marques et al., 2002a). A diversidade de uma biota insular pode variar de acordo com a área da ilha, o isolamento geográfico, sua idade de formação, a distância entre o continente e/ou ilhas vizinhas, a diversidade de habitats e a riqueza da fonte colonizadora (Darwin, 1859; MacArthur, Wilson, 1963; Connor, McCoy, 1979; Carbonari, 1981; Cox, Moore, 1993). Portanto, por possuírem maior diversidade de habitats, ilhas de grande porte tendem a apresentar maior riqueza e estabilidade em relação às menores, assim como as ilhas mais próximas de áreas continentais (MacArthur, Wilson, 1967). As ilhas apresentam menor número de espécies do que uma área de tamanho similar no continente, e geralmente apresentam populações diferenciadas, o que pode ser resultante da variação geográfica no clima, recursos alimentares, competição inter e intra-específica e intensidade de predação (ver Dunham et al., 1978; Case, 1978; King, 1989). Porém, apesar de sua baixa riqueza de espécies, ilhas geralmente são caracterizadas pela grande proporção de espécies endêmicas (Darwin, 1859).

O litoral do estado de São Paulo possui 129 formações insulares, incluindo ilhas e ilhotas, cuja maioria apresenta dimensões reduzidas e formação variada (Ângelo, 1989). Praticamente não há estudos abrangentes sobre a herpetofauna das ilhas do litoral paulista. Trabalhos desenvolvidos em ambientes insulares incluem as ilhas dos Alcatrazes (e.g. Amaral, 1921; Luederwaldt, Fonseca, 1923; Marques et al., 2002a), Queimada Grande (e.g. Hoge, 1950; Duarte et al., 1995; Marques et al., 2002b), Ilha do Mar Virado (Vieitas, 1995), Ilha Anchieta (Cicchi, 2007), Ilha do Cardoso (Sena, 2007) e Ilha de São Sebastião (e.g. Ihering, 1897; Müller, 1968; Luederwaldt, 1929; Sawaya, 1999; Centeno et al., 2008).

A Ilha de São Sebastião, localizada no litoral norte do estado de São Paulo, é a maior ilha continental do Brasil e apresenta cerca de 33.000 ha de Mata Atlântica, dos quais 27.000 são protegidos pelo Parque Estadual de Ilhabela (PEIB; SMA, 1998). Entretanto, apesar de parcialmente protegida por leis e programas de conservação, não se encontra fora dos perigos de impactos variados. A fragmentação florestal é conhecida como uma das principais causas da redução da diversidade biológica, e pode provocar declínios populacionais e extinções locais (cf. Brooks et al., 2002). Assim, a Ilha de São 
Sebastião apresenta grande importância para pesquisas detalhadas sobre a herpetofauna. Além disso, preservar os ambientes insulares deve ser tarefa prioritária nos programas de conservação da natureza (Ângelo, 1989). Devido à fragilidade de sua dinâmica, esses ecossistemas necessitam de normas específicas e restritas de preservação. A estratégia de conservação deveria tratar de forma diferente os ambientes insulares em relação aos continentais (Diamond, 1976), já que ilhas sofrem grande pressão antrópica, com conseqüências desastrosas para a diversidade biológica (Vitousek et al., 1995).

Considerando que a Ilha de São Sebastião está submetida a intensas alterações antrópicas, o conhecimento da diversidade e história natural de suas espécies é fundamental para o planejamento e decisões sobre estratégias de conservação. Este projeto teve como objetivo o estudo da herpetofauna da Ilha de São Sebastião, enfocando a composição, riqueza, dominância e abundância relativa de espécies, assim como informações sobre história natural, incluindo o uso do ambiente e do substrato. 


\section{CONCLUSÕES}

A Ilha de São Sebastião apresenta uma herpetofauna rica e singular, com pelo menos 35 espécies de anfíbios e 38 de répteis.

Entre os cinco métodos de amostragem utilizados, armadilhas de interceptação e queda apresentaram o melhor desempenho na captura de indivíduos. Em relação à riqueza de espécies, procura visual limitada por tempo e encontro casual apresentaram o melhor desempenho na captura de anfíbios e armadilha de interceptação e queda na captura de répteis.

Os métodos de amostragem utilizados neste estudo são complementares, já que apresentam limitações e dificilmente capturariam sozinhos a diversidade total de anfíbios e répteis da Ilha de São Sebastião.

Na Ilha de São Sebastião, Leptodactylus cf. marmoratus é espécie dominante entre os anfíbios (30,8\%) e Bothrops jararaca entre os répteis Squamata (29,5\%).

A composição de espécies da Ilha de São Sebastião é mais relacionada a taxocenoses de localidades de maior proximidade geográfica. Além da distância geográfica, fatores como insularização, relação espécie-área e degradação ambiental parecem estar envolvidos na estruturação dessas taxocenoses.

O modo como as espécies da herpetofauna da Ilha de São Sebastião utilizam o substrato é bastante diversificado, com maior número de espécies especialistas em relação a generalistas.

O substrato mais utilizado pela herpetofauna foi a serapilheira (25 spp.), seguida de arbusto (18 spp.), solo (17 spp.), riacho (12 spp.) e árvore (10 spp.).

Maior diversidade ocorreu na altitude intermediária (300 m), o que corresponde à maior riqueza e menor dominância.

A maior semelhança biogeográfica foi encontrada entre as faixas altitudinais de 300 e $600 \mathrm{~m}$. 
Entre as altitudes amostradas, houve uma tendência de diminuição no número de espécies com o aumento da altitude, com substituição da espécie dominante.

A Ilha de São Sebastião é extremamente importante para pesquisa e conservação, por apresentar uma fauna rica, diversificada, incluindo espécies endêmicas. 


\section{CONSIDERAÇÕES FINAIS}

A herpetofauna de Mata Atlântica na Ilha de São Sebastião é bastante diversificada com 73 espécies registradas até o momento. Entre as espécies registradas, pelo menos uma é endêmica da ilha (Siphonops insulanus), três estão na lista de espécies ameaçadas de extinção (Chiasmocleis carvalhoi, Mabuya caissara e Stereocyclops parkeri; Diário Oficial, 2008), uma se enquadra na categoria vulnerável (Hydromedusa maximiliani; IUCN, 2008) e seis possivelmente são espécies ainda não descritas (Brachycephalus aff. nodoterga, Ischnocnema sp. 1 e 2 (gr. lactea), Scinax aff. catharinae, S. aff. perpusillus, e Liotyphlops sp.).

Esta grande riqueza de espécies pode ser um reflexo das boas condições de preservação da vegetação encontrada na Ilha de São Sebastião, apesar de apresentar cerca de 30\% de sua vegetação alterada (Luederwaldt, 1929; França, 1954; Olmos, 1996). Como resultado de 500 anos de destruição, a Mata Atlântica sofreu forte degradação de sua cobertura vegetal em resposta a intensa pressão antrópica (Morellato, Haddad, 2000). Entretanto, é na Serra do Mar que se encontra a parcela mais representativa do que restou (SOS Mata Atlântica, 2002). A Ilha de São Sebastião está situada neste grande remanescente, sendo uma das áreas prioritárias para a conservação dentro da Mata Atlântica.

A Ilha de São Sebastião é uma das poucas ilhas protegidas por um parque estadual e, apesar de parcialmente protegida por leis e programas de conservação, não se encontra fora dos perigos de impactos ecológicos em seu ambiente nativo. Sabe-se que a fragmentação florestal é uma das principais causas da redução da diversidade biológica, que pode em longo prazo provocar declínios populacionais e extinções locais. Diante deste quadro, a Ilha de São Sebastião é de extrema importância para a conservação por abrigar uma fauna rica e diversificada, incluindo espécies endêmicas e ameaçadas de extinção. Além disso, apresenta grande potencial para a manutenção da herpetofauna da Mata Atlântica da região. 


\section{REFERÊNCIAS BIBLIOGRÁFICAS ${ }^{*}$}

Ab’Saber AN. Os domínios morfoclimáticos na América do Sul. Primeira aproximação. Geomorfologia. 1977;52: 1-21.

Amaral A. Contribuição para o conhecimento dos ophídios do Brasil. A. Parte II. Biologia de uma nova espécie. Anexos Mem Inst Butantan. 1921;1:39-44.

Ângelo S. Ilhas do litoral paulista. Série documentos. São Paulo: Secretaria do Meio Ambiente - SMA; 1989.

Begon M, Harper JL, Townsend CR. Ecology: individuals, populations and communities. Oxford: Blackwell Science; 2006.

Bertolotto CEV. Enyalius (Leiosauridae, Squamata): o que os dados moleculares e cromossômicos revelam sobre esse gênero de lagartos endêmico do Brasil [dissertação]. São Paulo: Instituto de Biociências da Universidade de São Paulo; 2006.

Bertoluci JA. Anfíbios anuros. In: Leonel, C, editor. Intervales: fundação para a conservação e produção florestal do estado de São Paulo. São Paulo: A Fundação; 2001. p.159-67.

Bertoluci J, Brassaloti RA, Ribeiro JW, Vilela V, Sawakuchi HO. Species composition and similarities among anuran assemblages of forest sites in southeastern Brazil. Sci agric. 2007;64(4):364-74.

Brandão RA, Araújo AFB. A herpetofauna da Estação Ecológica de Águas Emendadas. In: Marinho-Filho J, Rodrigues F, Guimarães M, editores. Vertebrados da Estação Ecológica de Águas Emendadas: história natural e ecologia em um fragmento de cerrado do Brasil central. Brasília: SEMATEC/IEMA; 1998. p. 9-21.

Brasileiro CA, Sawaya, RJ, Kiefer MC, Martins M. Amphibians of an open cerrado fragment in southeastern Brazil. Biota Neotrop. 2005;5(2):1-17.

Brooks TM, Mittermeier RA, Mittermeier CG, Da Fonseca GAB, Rylands AB, Konstant WR, Flick P, Pilgrim J, Oldfield S, Magin G, Hilton-Taylor C. Habitat Loss and extinction in the hotspots of biodiversity. Conserv Biol. 2002;16(4):909-23.

Brown JH, Lomolino MV. Biogeografia. 2. ed. Ribeirão Preto: FUNPEC; 2006.

Burke VJ, Gibbons JW. Terrestrial buffer zones and wetland conservation: a case study of freshwater turtles in a Carolina bay. Conserv Biol. 1995;9(6):1365-9.

\footnotetext{
De acordo com:

International Committee of Medical Journal Editors. Uniform requirements for manuscripts submitted to Biomedical Journal: sample references. Available from: http://www.icmje.org [2007 May 22].
} 
Cadle JE, Greene HW. Phylogenetic patterns, biogeography, and the ecological structure of Neotropical snake assemblages. In: Ricklefs RE, Schluter D, editors. Species diversity in ecological communities: historical and geographical perspectives. Chicago: University of Chicago Press; 1993. p. 281-93.

Cadle JE, Patton JL. Distribution patterns of some amphibians, reptiles, and mammals of the eastern Andean slope of southern Peru. In: Vanzolini PE, Heyer WR, editors. Proceedings of a Workshop on Neotropical Distribution Patterns. Rio de Janeiro: Academia Brasileira de Ciências; 1988. p. 225-44.

Campbell HW, Christman SP. Field techniques for herpetofaunal community analysis. In: Scott Junior NJ, editor. Herpetological communities: a symposium of the Society for the Study of Amphibians and Reptiles and the Herpetologist's League. U S Fish and Wildlife Service Wildlife Research Report. 1982;13:193-200.

Carbonari MP. Caderno de ciências da Terra. Ecossistema Insular: importância de seu estudo. São Paulo: Universidade de São Paulo; 1981.

Cardoso AJ, Andrade GV, Haddad CFB. Distribuição espacial em comunidades de anfíbios (Anura) no Sudeste do Brasil. Rev Bras Biol. 1989;49(1):241-50.

Case TJ. A general explanation for insular body size trends in terrestrial vertebrates. Ecology. 1978;59(1):1-18.

Cechin SZ. História natural de uma comunidade de serpentes na região da depressão central no Rio Grande do Sul, Brasil [tese]. Porto Alegre: Faculdade de Biociências da Pontifícia Universidade Católica do Rio Grande do Sul; 1999.

Cechin SZ, Martins M. Eficiência de armadilhas de queda (pitfall traps) em amostragens de anfíbios e répteis no Brasil. Rev Bras Zool. 2000;17(3):729-40.

Centeno FC, Sawaya RJ, Marques OAV. Snake assemblage of Ilha de São Sebastião, southeastern Brazil: comparison to mainland. Biota Neotrop. 2008;8(3):63-8.

CEPAGRI. Centro de Pesquisa Meteorológica e Climática Aplicada à Agricultura: Clima dos municípios paulistas. Disponível em http://www.cpa.unicamp.br [2008 set].

Cicchi PJP. Dados ecológicos da herpetofauna do Parque Estadual da Ilha Anchieta, Ubatuba, São Paulo, Brasil [dissertação]. Botucatu: Instituto de Biociências da Universidade Estadual Paulista; 2007.

Cicchi PJP, De Sena MA, Peccinini-Seale DM, Duarte MR. Serpentes das ilhas costeiras do estado de São Paulo, sudeste do Brasil. Biota Neotrop. 2007;7(2): 227-40.

Colli GR, Peres AK, da Cunha HJ. A new species of Tupinambis (Squamata: Teiidae) from central Brazil, with an analysis of morphological and genetic variation in the genus. Herpetologica. 1998;54(4):477-92. 
Colwell RK. EstimateS (version 7.5) - Statistical estimation of species richness and shared species from samples. University of Connecticut. http://viceroy.eeb.uconn.edu/estimates, 2005.

Connor EF, McCoy ED. The statistics and biology of the species-area relationship. Am Nat. 1979;113(6):791-833.

Conte CE, Rossa-Feres DD. Diversidade e ocorrência temporal da anurofauna (Anura: Amphibia) em São José dos Pinhais, Paraná, Brasil. Revista Brasileira De Zoologia. 2006;23(1):162-75.

Corn PS. Straight-line drift fences and pitfall traps. In: Heyer WR, Donnely MA, McDiarmid RW, Hayek LA, Foster M, editors. Measuring and monitoring biological biodiversity: standard methods for amphibians. Washington DC: Smithsonian Institution Press; 1994. p. 118-24.

Cox CB, Moore PD. Biogeography: an ecological and evolutionary approach. Oxford: Blackwell Science; 1993.

Crawford JA, Semlitsch RD. Abiotic factors influencing abundance and microhabitat use of stream salamanders in southern Appalachian forests. For Ecol Manage. 2008;255(5-6):1841-7.

Crosswhite DL, Fox SF, Thill RE. Comparison of methods for monitoring reptiles and amphibians in upland forests of the Ouachita Mountains. Proc Oklahoma Acad Sci. 1999;79:45-50.

Crump ML, Scott Junior NJ. Visual encounter surveys. In: Heyer WR, Donnely MA, McDiarmid RW, Hayek LA, Foster M, editors. Measuring and monitoring biological biodiversity: standard methods for amphibians. Washington DC: Smithsonian Institution Press; 1994, p. 84-92.

Da Fonseca GAB, Rylands A, Paglia A, Mittermeier RA. Atlantic Forest. In: Mittermeier RA, Gil PR, Hoffman M, Pilgrim J, Brooks T, Mittermeier CG, Lamoreux J, Da Fonseca GAB, organizers. Hotspots revisited: earth's biologically richest and most endangered terrestrial ecoregions. CEMEX: Agrupación Sierra Madre; 2005.

Darwin CR. On the origin of species by means of natural selection. 8th ed. London: Oxford University Press; 1859.

Diamond JM. Island biogeography and conservation: strategy and limitations. Science. 1976;193:1027-1029.

Diário Oficial. Estado de São Paulo. Decreto N ${ }^{\circ}$ 53.494, de 2 de outubro de 2008, 118(187), São Paulo; 2008.

Di-Bernardo M. História Natural de uma comunidade de serpentes da borda oriental do planalto das Araucárias Rio Grande do Sul Brasil [tese]. Rio Claro: Instituto de Biociências da Universidade Estadual Paulista; 1998. 
Dixo M, Verdade VK. Leaf litter herpetofauna of the Reserva Florestal de Morro Grande, Cotia (SP). Biota Neotrop. 2006;6(2):1-20.

Dixon JR, Kofron CP. The Central and South American anomalepid snakes of the genus Liotyphlops. Amphibia-Reptilia. 1983;4(2-4):241-64.

Domenico EA. Herpetofauna do Mosaico de Unidades de Conservação do Jacupiranga (SP) [dissertação]. São Paulo: Instituto de Biociências da Universidade de São Paulo; 2008.

Duarte MR, Puorto G, Franco FL. A biological survey of the pitviper Bothrops insularis Amaral (Serpentes, Viperidae): an endemic and threatened offshore island snake of southeastern Brazil. Stud Neotrop Fauna Environm. 1995;30(1):1-13.

Duellman WE. The biology of an equatorial herpetofauna in Amazonian Ecuador. University of Kansas, Museum of Natural History, Miscellaneous Publication. 1978(65):1-352.

Duellman WE. Patterns of species-diversity in anuran amphibians in the american tropics. Ann Missouri Bot Gard. 1988;75(1):79-104.

Duellman WE. Tropical herpetofaunal communities: patterns of community structure in Neotropical rainforests. In: Harmelin-Vivien M, Bourliere F, editors. Ecological studies, vol 69, vertebrates in complex tropical systems. New York: Springer-Verlag; 1989. p. 61-88.

Duellman WE, Gentry AH. Herpetofaunas in neotropical rainforests: comparative composition, history, and resource use. In: Gentry AH, editor. Four neotropical rainforests. New Haven: Yale University Press; 1990. p. 455-505.

Duellman WE, Trueb L. Biology of amphibians. New York: McGraw-Hill Book Company; 1994.

Dunham AE, Tinkle DW, Gibbons JW. Body size in islands lizards: a cautionary tale. Ecology. 1978;59(6):1230-1238.

Estes R, Queiroz K, Gauthier J, Pregill G. Phylogenetic relationships within the Squamata. In: Estes R, Pregill G, editors. Phylogenetic relationships of the lizard families. Essays commemorating Charles L Camp. California: Standford; 1988. p. 119281.

Faivovich J, Haddad CFB, Garcia PCA, Frost DR, Campbell JA, Wheeler WC. Systematic review of the frog family hylidae, with special reference to hylinae: phylogenetic analysis and taxonomic revision. Bull Am Mus Nat Hist. 2005;(294):6228.

Fauth JE, Crother BI, Slowinski JB. Elevational patterns of species richness, evenness, and abundance of the Costa Rican leaf-litter herpetofauna. Biotropica. 1989;21(2):17885. 
Ferrarezzi H. Uma sinopse dos gêneros e classificação das Serpentes (Squamata): II. família Colubridae. In: Oliveira ME, Barreto L, editores. Herpetologia no Brasil. Belo Horizonte: Sociedade Brasileira de Herpetologia; 1994. p. 81-91.

França A. Notas sobre a geografia da Ilha de S. Sebastião. Bol Assoc Geogr Bras. 1944;4(5):49-59.

França A. A Ilha de São Sebastião: estudo de geografia humana. São Paulo: Universidade de São Paulo, Faculdade de Filosofia, Ciências e Letras. Boletim 178, Geografia n.10; 1954.

Freire EMX, Caramaschi U, Argolo AJS. A new species of Liotyphlops (Serpentes: Anomalepididae) from the Atlantic Rain Forest of Northeastern Brazil. Zootaxa. 2007;(1393):19-26.

Freitas RO. Geomorfogênese da Ilha de São Sebastião. Bol Assoc Geogr Bras. 1944a;4(4):16-30.

Freitas RO. Jazimento das rochas alcalinas no Brasil meridional. Mineração e Metalurgia. 1944b;8(43): 45-48.

Freitas RO. Geologia e petrologia da Ilha de São Sebastião. Boletim FFLCH/USP. 1947;85(3):1-244.

Frost DR. Amphibian species of the world 5.1, an online reference. Available from: http://research.amnh.org/herpetology/amphibia/index.php [2008 Oct].

Giaretta AA. Diversidade e densidade de anuros de serapilheira num gradiente altitudinal na Mata Atlântica costeira [tese]. Campinas: Instituto de Biologia da Universidade Estadual de Campinas; 1999.

Gotelli NJ, Colwell RK. Quantifying biodiversity: procedures and pitfalls in the measurement and comparison of species richness. Ecol Lett. 2001;4(4):379-91.

Gotelli NJ, Entsminger GL. EcoSim (Version 7.0.) - Null models software for ecology. Acquired Intelligence Inc., Kesey-Bear. http://homepages.together.net/ gentsmin/ecosim.htm; 2005.

Greenberg CH, Neary DG, Harris LD. A comparison of herpetofaunal sampling effectiveness of pitfall, single-ended, and double-ended funnel traps used with drift fences. J Herpetol. 1994;28(3):319-24.

Haddad CFB, Sazima I. Anfíbios anuros da Serra do Japi. In: Morellato LPC, organizador. História Natural da Serra do Japi: ecologia e preservação de uma área florestal no sudeste do Brasil. Campinas: FAPESP e Editora da Universidade Estadual de Campinas; 1992. p. 188-211.

Haddad CFB, Toledo LF, Prado CPA. Anfíbios da Mata Atlântica: guia dos anfíbios anuros da Mata Atlântica. São Paulo: Editora Neotropica; 2008. 
Hartmann MT. Biologia reprodutiva de uma comunidade de anuros (Amphibia) na Mata Atlântica (Picinguaba, Ubatuba, SP) [tese]. Rio Claro: Instituto de Biociências da Universidade Estadual Paulista “Júlio de Mesquita Filho”; 2004.

Hartmann PA. História natural e ecologia de duas taxocenoses de serpentes na Mata Atlântica [tese]. Rio Claro: Instituto de Biociências da Universidade Estadual Paulista "Júlio de Mesquita Filho"; 2005.

Henderson RW, Dixon JR, Soini P. Resource partitioning in Amazonian Peru snake communities. Milwaukee Public, Museum Contributions in Biology and Geology. 1979;(22):1-12.

Hennies WT, Hasui Y. Geocronologia das rochas eruptivas da Ilha de São Sebastião, SP. Anais do Congresso Brasileiro de Geologia. 1968;22:145-148.

Hennies WT, Hasui Y. Contribuição ao reconhecimento da geologia da Ilha de São Sebastião. Atas do Simpósio de Geologia Regional. 1977;1:199-209.

Heyer WR. On frog distribution patterns east of the Andes. In: Vanzolini PE, Heyer WR, editors. Proceedings of a Workshop on neotropical distribution patterns. Rio de Janeiro: Academia Brasileira de Ciências; 1988. p. 245-273.

Heyer WR, Rand AS, Cruz CAGD, Peixoto OL, Nelson CE. Frogs of Boraceia. Arq Zool. 1990;31(4):231-410.

Hofer U, Bersier LF. Herpetofaunal diversity and abundance in tropical upland forests of cameroon and panama. Biotropica. 2001;33(1):142-52.

Hoge AR. Notas erpetológicas 7. Fauna erpetológica da Ilha de Queimada Grande. Memo Inst Butantan. 1950;22:151-172.

Ihering HV. A Ilha de S. Sebastião. Rev Mus Paul. 1897;129-71.

Ingram GB. The remaining islands with primary rain-forest: a global resource. Environ Manage. 1992;16(5):585-95.

IUCN. 2008 IUCN Red List of Threatened Species. Available from: http://www.iucnredlist.org. 2008 [2008 Oct 16].

Janzen DH. Sweep samples of tropical foliage insects: description of study sites, with data on species abundances and size distributions. Ecology. 1973;54(3):659-86.

Jim J. Aspectos gerais da anurofauna da região de Botucatu. In: Uieda W, Paleari LM, organizadores. Fauna e flora: um dossiê ambiental. São Paulo: Editora da UNESP; 2004, p. 75-89.

King RB. Body size variation among island and mainland snake populations. Herpetologica. 1989;45(1):84-88.

Krebs CJ. Ecological methodology. New York: Harper and Row, Publishers; 1989. 
Kovach WL. MVSP - A multi-variate statistical package for windows, versão. 3.1. Kovach computing Services, Penthraeth; 1999.

Liddle MJ, Scorgie HRA. The effects of recreation on fresh-water plants and animals: a review. Biol Conserv. 1980;17(3):183-206.

Lieberman D, Lieberman M, Peralta R, Hartshorn GS. Tropical forest structure and composition on a large-scale altitudinal gradient in Costa Rica. J Ecology. 1996;84(2):137-52.

Lima AP, Magnusson WE, Menin M, Erdtmann LK, Rodrigues DJ, Keller C, Hödl W. Guia dos Sapos da Reserva Adolpho Ducke, Amazônia Central. Manaus: Attama; 2006.

Lino CF. Reserva da biosfera da Mata Atlântica. Plano de ação, vol.1, referências básicas. Campinas: Universidade Estadual de Campinas, 1992.

Loehle C, Wigley TB, Shipman PA, Fox SF, Rutzmoser S, Thill RE, Melchiors MA. Herpetofaunal species richness responses to forest landscape structure in Arkansas. For Ecol Manage. 2005;209(3):293-308.

Losos JB. Phylogenetic perspectives on community ecology. Ecology. 1996;77(5):1344-54.

Lucas EM, Fortes VB. Diversidade de anuros na Floresta Nacional de Chapecó, Floresta Atlântica do sul do Brasil. Biota Neotrop. 2008;8(3):51-61.

Luederwaldt H. Resultados de uma excursão científica à Ilha de S. Sebastião em 1925. Rev Mus Paul. 1929;16:38-40.

Luederwaldt H, Fonseca JP. A Ilha de Alcatrazes. Rev Mus Paul. 1923;13:441-512.

MacArthur RH, Wilson EO. An equilibrium theory of insular zoogeography. Evolution. 1963;17(4):373-87.

MacArthur RH, Wilson EO. The theory of island biogeography. Princeton: Princeton University Press; 1967.

Magurran AE. Measuring biological diversity. Oxford: Blackwell Science; 2004.

Marques OAV. Composição faunística, história natural e ecologia de serpentes da Mata Atlântica, na região da Estação Ecológica Juréia-Itatins, São Paulo, SP [tese]. São Paulo: Instituto de Biociências da Universidade de São Paulo; 1998.

Marques OAV, Eterovick A, Sazima I. Serpentes da Mata Atlântica: guia ilustrado para a Serra do Mar. Ribeirão Preto: Holos; 2001.

Marques OAV, Martins M, Sazima I. A jararaca da Ilha da Queimada Grande. Ciência Hoje. 2002a;31(186):56-9. 
Marques OAV, Martins M, Sazima I. A new insular species of pitviper from Brazil, with comments on evolutionary biology and conservation of the Bothrops jararaca group (Serpentes, Viperidae). Herpetologica. 2002b;58(3):303-12.

Marques OAV, Sazima I. História natural dos répteis da Estação Ecológica JuréiaItatins. In: Marques OAV, Duleba W, editores. Estação Ecológica Juréia-Itatins: ambiente físico, flora e fauna. Ribeirão Preto: Holos Editora; 2004. p. 257-77.

Martins MRC. História natural e ecologia de uma taxocenose de serpentes de mata na região de Manaus, Amazônia Central, Brasil [tese]. Campinas: Instituto de Biologia da Universidade Estadual de Campinas; 1994.

Martins M, Oliveira ME. Natural history of snakes in forests of the Manaus region, central Amazonia, Brazil. Herpetol Nat Hist. 1998;6(2):78-150.

Mattos JNB. O Clima de São Paulo. Secretaria da Agricultura, Commercio e Obras Públicas do Estado de São Paulo. Serviço Metereologico. São Paulo; 1925. (Série II, n.38).

McCoy ED. The distribution of insects along elevational gradients. Oikos. 1990;58(3):313-22.

Melo AS, Pereira RAS, Santos AJ, Shepherd GJ, Machado G, Medeiros HF, Sawaya RJ. Comparing species richness among assemblages using sample units: why not use extrapolation methods to standardize different sample sizes? Oikos. 2003;101(2):398410.

Milliman JD, Emery KO. Sea levels during past 35000 years. Science. 1968;162(3858):1121-23.

Milanesi MA. Avaliação do efeito orográfico na pluviometria de vertentes opostas da Ilha de São Sebastião (Ilhabela - SP) [dissertação]. São Paulo: Faculdade de Filosofia, Letras e Ciências Humanas, Departamento de Geografia da Universidade de São Paulo; 2007.

Ministério do Meio Ambiente. Diretrizes para a política de conservação e desenvolvimento sustentável da Mata Atlântica. Brasília: MMA; 1999. (Caderno, n.13).

Moraes RA, Sawaya RJ, Barrella W. Composição e diversidade de anfíbios anuros em dois ambientes de Mata Atlântica no Parque Estadual Carlos Botelho, São Paulo, sudeste do Brasil. Biota Neotrop. 2007;7(2):1-10.

Morellato LPC, Haddad CFB. Introduction: The Brazilian Atlantic Forest. Biotropica. 2000;32(4B):786-92.

Müller, P. Die herpetofauna der Insel von São Sebastião (Brasilien). Germany: Saarbrücken Zeitung, Verlag und Druckerei GmbH; 1968.

Murphy DD. Desafios à diversidade biológica em áreas urbanas. In: Wilson EO, organizador. Biodiversidade. Rio de Janeiro: Nova Fronteira; 1997. 
Myers N, Mittermeier RA, Mittermeier CG, da Fonseca GAB, Kent J. Biodiversity hotspots for conservation priorities. Nature. 2000;403(6772):853-8.

Nascimento CM. Atlas climatológico do Estado de São Paulo (1977-1986). Campinas: Fundação Cargill; 1988.

Nimer E. Climatologia do Brasil. 2. ed. Rio de Janeiro: IBGE, Departamento de Recursos Naturais e Estudos Ambientais; 1989.

Olmos F. Missing species in São Sebastião Island, southeastern Brazil. Pap Avulsos Zool. 1996;39(18):329-49.

Olson DM. The distribution of leaf-litter invertebrates along a neotropical altitudinal gradient. J Trop Ecol. 1994;10:129-50.

Owen JG. Patterns of herpetofaunal species richness: relation to temperature, precipitation, and variance in elevation. J Biogeogr. 1989;16(2):141-50.

Pavan D. Assembléias de répteis e anfíbios do Cerrado ao longo da bacia do rio Tocantins e o impacto do aproveitamento hidrelétrico da região na sua conservação [tese]. São Paulo: Instituto de Biociências da Universidade de São Paulo; 2007.

Pearman PB. Correlates of amphibian diversity in an altered landscape of Amazonian Ecuador. Conserv Biol. 1997;11(5):1211-25.

Pianka ER. Evolutionary ecology. 5th ed. New York: Harper Collins; 1994.

Pombal Junior JP. Biologia reprodutiva de anuros (Amphibia) associados a uma poça permanente na Serra de Paranapiacaba, sudeste do Brasil [tese]. Rio Claro: Instituto de Biociências da Universidade Estadual Paulista “Júlio de Mesquita Filho”; 1995.

Pombal Junior JP, Gordo M. Anfíbios anuros da Juréia. In: Marques OAV, Duleba W, editores. Estação Ecológica Juréia-Itatins: ambiente físico, flora e fauna. Ribeirão Preto: Holos Editora; 2004. p. 243-56.

Pough FH, Andrews RM, Cadle JE, Crump ML, Savitzky AH, Wells KD. Herpetology. 3rd ed. New Jersey: Pearson Prentice-Hall; 2004.

Rahbek C. The relationship among area, elevation, and regional species richness in neotropical birds. Am Nat. 1997;149(5):875-902.

Rebouças-Spieker R. Distribution and differentiation of animals along the coast and on continental islands of the state of São Paulo, Brasil. 2. Lizards of the genus Mabuya (Sauria, Scincidae). Pap Avul Zool. 1974;28(12):197-240.

Ribeiro RDS, Egito GTBT, Haddad CFB. Chave de identificação: anfíbios anuros da vertente de Jundiaí da Serra do Japi, estado de São Paulo. Biota Neotrop. 2005;5(2):115.

Ricklefs RE. Ecology. New York: Freeman and Company; 1990. 
Rocha CFD, Van Sluys M, Bergallo HG, Alves MAS. Endemic and threatened tetrapods in the restingas of the biodiversity corridors of Serra do Mar and of the Central da Mata Atlantica in eastern Brazil. Braz J Biol. 2005;65(1):159-68.

Rodrigues MT. The conservation of Brazilian reptiles: challenges for a megadiverse country. Conserv Biol. 2005;19(3):659-64.

Rossa-Feres DC, Martins M, Marques OAV; Martins IA, Sawaya RJ, Haddad CFB. Herpetofauna. In: Rodrigues RR et al., coordenadores. Diretrizes para a conservação e restauração da biodiversidade no estado de São Paulo. São Paulo: Instituto de Botânica, FAPESP e Programa BIOTA/FAPESP, p. 82,94. No prelo 2008.

Samson DA, Rickart EA, Gonzales PC. Ant diversity and abundance along an elevational gradient in the Philippines. Biotropica. 1997;29(3):349-63.

Sanders HL. Marine benthic diversity: a comparative study. Am Nat. 1968;102(925):243-82.

Sawaya RJ. Diversidade, densidade e distribuição altitudinal da anurofauna de serapilheira da Ilha de São Sebastião, SP [dissertação]. São Paulo: Instituto de Biociências da Universidade de São Paulo; 1999.

Sawaya RJ. História natural e ecologia das serpentes de cerrado da região de Itirapina, SP [tese]. Campinas: Instituto de Biologia da Universidade Estadual de Campinas; 2004.

Sawaya RJ, Haddad CFB. Amphibia, Anura, Stereocyclops parkeri: distribution extention, new state record, geographic distribution map. Check List. 2004;2:74-76.

Sawaya RJ, Marques OAV, Martins M. Composição e história natural das serpentes de Cerrado de Itirapina, São Paulo, sudeste do Brasil. Biota Neotrop. 2008;8(2):127-49.

Sazima I, Répteis. In: Leonel C, editor. Intervales: fundação para a conservação e produção florestal do estado de São Paulo. São Paulo: A Fundação; 2001. p. 147-57.

Sazima I, Haddad CFB. Répteis da Serra do Japi: notas sobre história natural. In: Morellato LPC, organizador. História natural da Serra do Japi: ecologia e preservação de uma área florestal no sudeste do Brasil. Campinas: FAPESP; Editora da Universidade Estadual de Campinas; 1992. p. 212-36.

SBH. Sociedade Brasileira de Herpetologia. Disponível em: HTTP://www.sbherpetologia.org.br [2008 abr 27].

Schlaepfer MA, Gavin TA. Edge effects on lizards and frogs in tropical forest fragments. Conserv Biol. 2001;15(4):1079-90.

Scott Junior NJ. The abundance and diversity of the herpetofaunas of tropical forest litter. Biotropica. 1976;8(1):41-58. 
Scott Junior NJ, Maxwell TC, Thornton OW, Fitzgerald LA, Flury JW. Distribution, habitat, and future of harters water snake, nerodia-harteri, in Texas. J Herpetol. 1989;23(4):373-89.

Secretaria do Meio Ambiente. Planos de manejo das unidades de conservação: Parque Estadual de Ilhabela - Fase 1, Plano de Gestão Ambiental. São Paulo: SMA; 1998. (Documentos Ambientais).

Semlitsch RD. Biological delineation of terrestrial buffer zones for pond-breeding salamanders. Conserv Biol. 1998;12(5):1113-9.

Sena MA. Levantamento da fauna e estudo cromossômico de algumas espécies de Reptilia, Squamata, do município de Cananéia, SP [dissertação]. São Paulo: Instituto de Biociências da Universidade de São Paulo; 2007.

Shine R. Habitats, diets, and sympatry in snakes: study from Australia. Can J Zool. 1977;55(7):1118-28.

Silva JF. Dados climatológicos de Cananéia e Ubatuba (Estado de São Paulo). Bol. Climatol Inst Oceanogr. 1989;6:1-21.

SOS Mata Atlântica. Evolução dos remanescentes florestais e ecossistemas associados do domínio Mata Atlântica no período 1995-2000. São Paulo; 2002.

StatSoft. Statistica (data analysis software system), version 6.1. Tulsa, USA; 2003.

Stout J, Vandermeer J. Comparison of species richness for stream-inhabiting insects in tropical and mid-latitude streams. Am Nat. 1975;109(967):263-80.

Strüssmann C. Serpentes do pantanal de Poconé, Mato Grosso: composição faunística, história natural e ecologia comparada [tese]. Campinas: Instituto de Biologia da Universidade Estadual de Campinas; 1992.

Suguio K, Martin L. Classificação de costas e evolução geológica das planícies litorâneas quaternárias do sudeste e sul do Brasil. Anais do Simpósio de Ecossistemas da Costa Sul e Sudeste do Brasil. 1987;1:1-28.

Thurow GR. Herpetofaunal changes in McDonough County, Illinois. Transactions of the Illinois State Acad Sci. 1999;92(1-2):147-61.

Toledo LF, Zina J, Haddad CFB. Temporal and spatial distribution in an anuran community in municipality of Rio Claro, SP, Brazil. Holos Environ. 2003;3(2):136-49.

Trenham PC, Shaffer HB. Amphibian upland habitat use and its consequences for population viability. Ecol Appl. 2005;15(4):1158-68.

Uetz P, Hallermann J. The tigr reptile database. Available from: http://www.reptiledatabase.org [2008 Apr 25]. 
Vanzolini PE. Distribution and differentiation of animals along the coast and in continental islands of the state of S. Paulo, Brasil. I. Introduction to the area and problems. Pap Avul Zool. 1973;26(24):281-294.

Vanzolini PE. Guiano-Brasilian Polychrus: distribution and speciation (Sauria: Iguanidae). In: Rhodin AGJ, Miyata $\mathrm{K}$, editors. Advances in herpetology and evolutionary biology. Essays in honor of Ernest E Williams. Museum of Comparative Zoology, Cambrigde, Massachussets; 1983. p. 118-31.

Vieitas C. Análise Ambiental das Ilhas Costeiras da região de Ubatuba (SP), situação conservacionista e proposta de manejo para a Ilha do Mar Virado [dissertação]. São Paulo: Universidade de São Paulo; 1995.

Vitousek PM, Loope LL, Andersen H. Islands: biological diversity and ecossystem function. Ecological studies 115. Berlin Heidelberg: Spring-Verlag; 1995.

Vitt L, Magnusson WE, Pires TCA, Lima AP. Guia de Lagartos da Reserva Adolpho Ducke, Amazônia Central. Manaus: Áttema Design Editorial; 2008.

Wilcox BA, Murphy DD. Conservation strategy: the effects of fragmentation on extinction. Am Nat. 1985;125(6):879-87.

Wolda H. Similarity indexes, sample-size and diversity. Oecologia. 1981;50(3):296302.

Zar JH. Biostatistical Analysis. New Jersey: Prentice-Hall; 1996. 